\title{
Developing Movement Efficiency Between 7 and 9 Years of Age
}

\author{
Ida Maria Bosga-Stork, Jurjen Bosga, \\ and Ruud G.J. Meulenbroek
}

\begin{abstract}
This longitudinal study examined the movement efficiency of typically developing children between 7 and 9 years of age by scrutinizing their movement amplitudes and frequencies as they settled into a loop-writing task in which both parameters were prescribed. It was hypothesized that during the first three grades at primary school children would show increasing efficiency in exploiting the inverse relationship between movement amplitude and frequency when adjusting their movement errors. Whereas a clear developmental trend showed increasing efficiency with respect to the way in which the primary school children met the amplitude constraints, a more variable pattern was found for the age-dependent adjustments to the frequency requirements. At the level of parameter-error corrections from one cycle to the next, a marginal developmental trend was observed. Results are discussed in terms of contrasting effects between educational targets and movement-efficiency principles.
\end{abstract}

Keywords: adaptation, handwriting, learning, motor control, motor development

The study of efficiency principles in human movement control has a long history. The systematic relationships that exist between spatial and temporal movement parameters have been captured in principles such as isochrony (Viviani \& Terzuelo, 1980; Viviani \& McCollum, 1983), isogony, the two-thirds power law (Laquaniti, Terzuelo, \& Viviani, 1984) and the speed-accuracy trade off known as Fitts's law (Fitts, 1954).

In many everyday motor tasks multiple goals need to be achieved simultaneously. For example, speed and accuracy always are competing task constraints even though they are inversely related. In the face of such conflicts, it would be effective if one could find an efficient way of serving multiple purposes simultaneously. Some repetitive motor tasks allow for such a strategy. In cyclical motor tasks that need to be performed at a prescribed amplitude and frequency, the actor can strategically focus on changing one of the parameters to keep its value within acceptable boundaries while allowing the other to co-vary passively (Bosga-Stork, Bosga, $\&$ Meulenbroek, 2011). Given people's limited processing capacity (Hazeltine

Bosga-Stork and Bosga are with the Praktijk Bosga-Stork, De Beaufortweg, Netherlands. Meulenbroek is with the Donders Institute for Brain, Cognition and Behaviour, Radboud University Nijmegen, Nijmegen, Netherlands. 
\& Wifall, 2011) such a strategy seems more efficient than trying to continuously monitor and adjust both parameters simultaneously.

In a recent study we investigated the error-correction strategies from one movement to the next that primary school children adopt in a cyclical handwriting task with prescribed amplitude-frequency combinations (Bosga-Stork et al. 2011). We found that young children in first grade (at the age of 7) were able to exploit the inverse relationship between amplitude and frequency that follows from Fitts's law, particularly when they were pressed for speed. The changes of the movement parameters during task performance were more frequent in line with the inverse relationship than not, indicating that children also adopted a strategy of changing one of the parameters to reach a target value and let the other parameter co-vary passively. This left the question unanswered as to how such performance strategy develops with age. To answer this question we asked in the present longitudinal study the same group of children in second and third grade (8 and 9 years of age), to perform the same loop-writing task with prescribed amplitude-frequency combinations as used when they were 7 years old (Bosga-Stork et al., 2011). To what extent do children manage to contain their movement errors within an acceptable range as they develop from preparatory writers into more experienced writers (Meulenbroek \& Van Galen, 1988)? How do they achieve such control?

We used the same target amplitude-frequency combinations as in our previous study (Bosga-Stork et al., 2011). The diagram of the design of our study depicted in Figure 1 shows the four different amplitude and three frequency conditions. The drawing performances over the two years of follow-up were recorded.

In the current study we analyzed our longitudinal data on two different time scales, thus both providing a window into global development and into local task adaptations (Thelen, 2005; Von Hofsten, 2004). Development, generally referring to long-term behavioral changes due to maturation, growth, and learning, is here studied in the first three years of primary school covering the period of handwriting development from preparatory to experienced handwriting. With task adaptation we refer to the way people locally alter an individual movement of a specific action sequence to accommodate the constraints of the task at hand (Magill, 2011; Morris, 2009; Shumway-Cook \& Woollacott, 2007). We presume that the child actively explores a given task and detects information to adjust his or her performance to achieve the task goals (Dusing \& Harbourne, 2010). We capture adaptation by assessing the kinematic adjustments to the amplitude and frequency demands on a cycle-to-cycle basis within the first few counterclockwise rotating loop patterns. A description of the categorization of the cycle-to-cycle parameter-error changes that we distinguished can be found in Bosga-Stork et al. (2011). We have summarized this categorization in Figure 2, which is described in detail in the Methods section. Suffice to restate here that we distinguished between efficient and inefficient movement-error corrections depending on whether the cycle-to-cycle parameter changes were in line with the inverse relationship between amplitude and frequency or not.

Two hypotheses were formulated. One of the results of our earlier study was that older first graders (differentiating between old and young pupils on birthday) produced smaller frequency errors as a result of stronger perception-action coupling (Bosga-Stork et al., 2011). Therefore, we expected that the children, when being reassessed in the second and third grades of primary school, would become gradually more skilled in exploiting the inverse relationship between movement 


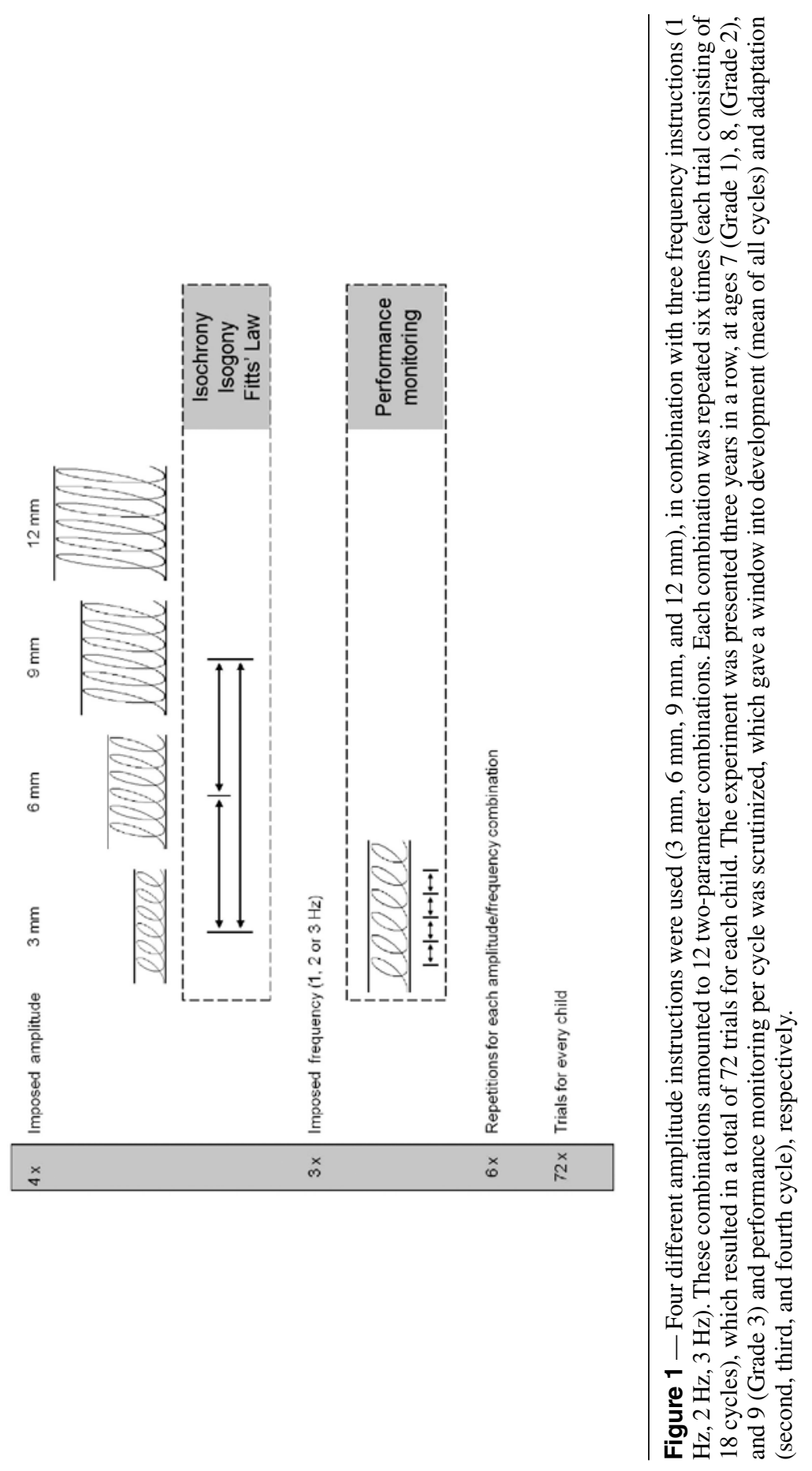




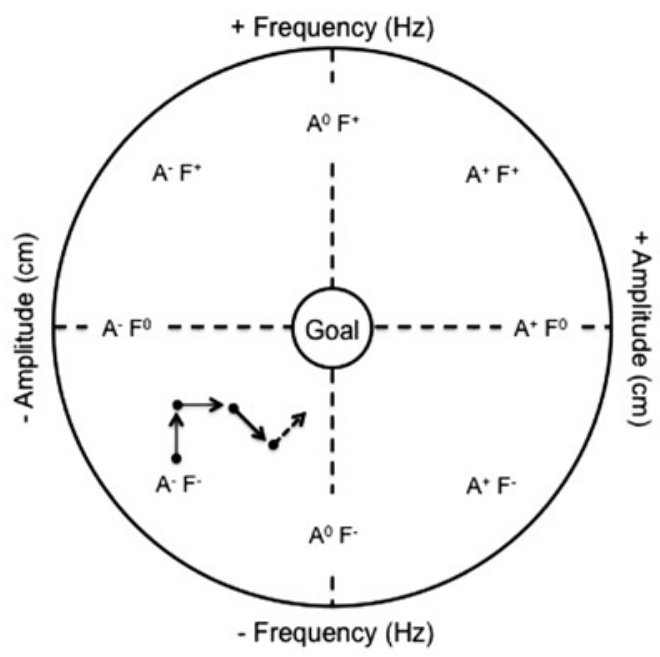

Figure 2 - Possible changes in performance in successive cycles. The sequence of the arrows toward the goal in the center depicts a hypothetical series of transitions in amplitudefrequency space, beginning with an amplitude that is too small (A-) and a frequency that is too low (F-). The first arrow pointing upwards represents a single parameter change (and error reduction) in the frequency domain; the second arrow pointing rightward represents a single parameter change (and error reduction) in the amplitude domain. The third arrow (bold) represents a double parameter change (of which the amplitude change is an error reduction and the frequency change an error increase) and the fourth arrow (bold dashed) indicates a quasi-double parameter change: both the amplitude and frequency errors are reduced and this compound error reduction corresponds with the inverse relationship that exists between amplitude and frequency.

amplitude and frequency (Newell \& Van Emmerik, 1989; Newell, 1986; Fitts \& Posner, 1967).

Secondly, with respect to how the children would contain their movement errors in the first part of the loop-writing task, we assumed that children, like adults, would settle into the task quickly, evidenced by decreasing performance errors in the second, third and fourth cycles (cf Figure 3 in. Bosga, Meulenbroek, \& Rosenbaum, 2005). Furthermore, we expected the children to increasingly exploit the inverse relationship between amplitude and frequency while adjusting their movement parameters on a cycle-to-cycle basis, as they develop from preparatory writers into more experienced writers

\section{Method}

\section{Participants}

A total of 34 typically developing primary school children were included in this study. At the start of this study they resided in two different 1st grade groups of two allied schools. The same cohort, with a fall out of two as result of change of schools, 


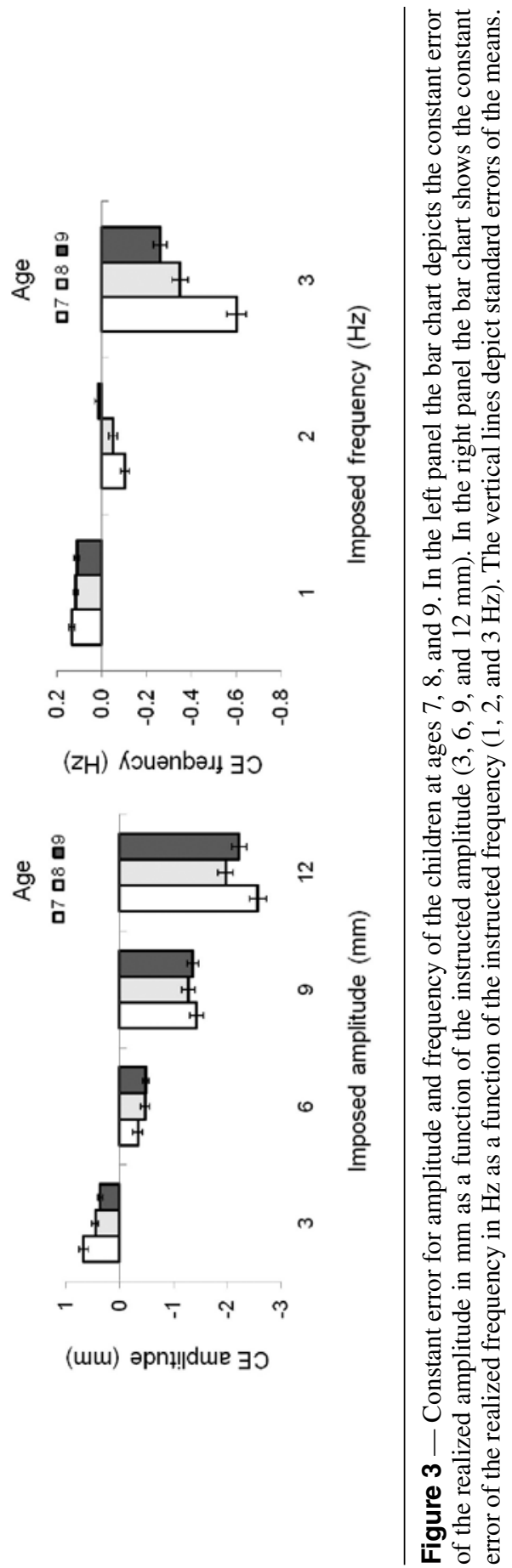


was followed over two years - starting as preparatory writers attending first grade, as first-year writers attending second grade, and as trained writers attending third grade. The group consisted of 15 girls and 17 boys with a mean age of 7.1 (range 6.4-7.6) in Grade 1. The children were tested each year in April. All participants had normal hearing and normal or corrected-to-normal vision. Each year all parents of the participants gave their informed consent. Each child received a little present after the experiment. Experimental procedures followed the APA guidelines for the ethical treatment of human participants.

\section{Procedure}

The experiment consisted of a writing task that was performed on a digitizer tablet. The same experiment was repeated twice in a 2-year time-span. The children were tested each year by the same experimenter (I.B-S, first author). The experiment took place in a separate, quiet, and well-lit room at the schools. The children were seated on an adjustable chair, with their feet supported and in a writing position adapted to the digitizer tablet. Participants were required to write loop patterns with an electronic ink pen (Intuos3) on preprinted sheets of paper attached to a digitizer tablet (WACOM A4 Oversize tablet). The loop patterns height was either within 3, 6,9 , or $12 \mathrm{~mm}$ lineation and the task was paced by an acoustic signal of either 1,2 , or $3 \mathrm{~Hz}$. The pacing signals changed sinusoidal in intensity across a clearly audible range (approximately $60-70 \mathrm{~dB}$; tone pitch $330 \mathrm{~Hz}$ ). Each of the 12 preprinted trial sheets consisted of six repetitions (block) of the twelve amplitude-frequency combinations and was presented at random. Amplitude-frequency combinations within the six trials of a block remained constant. Each child was asked to perform 72 trials of 18 loops each, leading to a theoretical total of 1296 loops per experiment at age 7, 8 and 9 (i.e., a maximum of 3888 loops per child). On-line recordings of $\mathrm{X}, \mathrm{Y}$ and $\mathrm{Z}$ (axial pen force) were sampled at $200 \mathrm{~Hz}$ (Bosga-Stork et al., 2011). Before the experiment started, the task was explained and the participants were allowed to perform the task a few times to get comfortable with experimental procedures and task requirements. For this purpose, each of the three frequencies was practice twice, using the 9 and $12 \mathrm{~mm}$ loop patterns, thus yielding 12 practice trials, in grade one. In grades two and three each of the three frequencies was practiced twice, using only the 9 -mm loop pattern (6 practice trials) since all children recognized the task and remembered what the procedure was.

\section{Data Analysis}

All data were visually inspected. After inspection, 224 trials $(1.06 \%)$ were rejected in the first experiment, 130 trials $(0.49 \%)$ in the second and 65 trials $(0.23 \%)$ in the third experiment. This was either due to missing data points when the children were taken by surprise by the pacing sound at the start of a trial or lagged behind the pacing signal too much during loop writing. To avoid artifacts due to smoothing by means of the Butterworth digital filter, we excluded the first and last cycle of each trial in the filtering. If the children were not able to comply with all 18 loops of a trial, the realized cycles of these trials were included. Execution of fewer cycles than instructed was either due to slowness in starting or executing a particular trial. In total 76,231 movement cycles (7 years: 21,$092 ; 8$ years: 26,669 and 9 years: 28,470 ) were evaluated in terms of realized amplitude and frequency relative to the 
goal amplitude and frequency, and with respect to the realized parameter change from one movement to the next. To allow zooming in on the settling-in process in each trial, we selected the second, third and fourth cycles in our error analyses ${ }^{1}$. The procedure of filtering the digitized pen-tip movements was identical to the one reported earlier (Bosga-Stork et al., 2011).

Amplitude and Frequency Errors The realized vertical amplitude (A expressed in $\mathrm{mm}$ ) and local cycle frequency ( $\mathrm{F}$ expressed in $\mathrm{Hz}$ ) were calculated for each writing loop. Following the procedure used earlier we calculated the local, signed parameter errors $\left(A_{\text {err }}\right.$ and $\left.F_{\text {err }}\right)$. The absolute error $(A E)$ of the amplitude and frequency $\left(\mathrm{AE}_{\mathrm{amp}}\right.$ and $\left.\mathrm{AE}_{\mathrm{frq}}\right)$ was calculated as the unsigned error expressed as a percentage of the instructed amplitude and frequency. Next, we calculated on the basis of the signed errors, the error changes from one cycle to the next. From each cycle, except the first, the two parameters $\Delta \mathrm{A}_{\mathrm{err}}$ and $\Delta \mathrm{F}_{\mathrm{err}}$ were determined where $\Delta \mathrm{A}_{\text {err }}$ equaled $\mathrm{A}_{\text {err }}$ of cycle i minus $\mathrm{A}_{\text {err }}$ of cycle $\mathrm{i}-1$, and $\Delta \mathrm{F}_{\text {err }}$ equaled $\mathrm{F}_{\text {err }}$ of cycle i minus $\mathrm{F}_{\text {err }}$ of cycle $\mathrm{i}-1$. A minimum value, $\mathrm{d}$, set at $1 \%$ of the locally instructed parameter value, was used to identify parameter changes. Any absolute value greater than or equal to this value qualified as a parameter change. We first categorized the $\mathrm{A}_{\mathrm{err}}$ and $\mathrm{F}_{\mathrm{err}}$ data into eight error types, which represented all possible combinations of overshoots and undershoots in the amplitude and frequency domain. Around a hypothetical goal eight possible performance errors might occur (see Figure 2). In a particular loop, a child can either make a single-parameter error (either amplitude or frequency is off-target: $\mathrm{A}^{0} \mathrm{~F}^{+}, \mathrm{A}^{+} \mathrm{F}^{0}, \mathrm{~A}^{0} \mathrm{~F}^{-}$and $\mathrm{A}^{-} \mathrm{F}^{0}$ ) or a double-parameter error (both amplitude and frequency are off-target: $\mathrm{A}^{+} \mathrm{F}^{+}, \mathrm{A}^{+} \mathrm{F}^{-}, \mathrm{A}^{-} \mathrm{F}^{-}$and $\mathrm{A}^{-} \mathrm{F}^{+}$).

The rationale for this classification is fully described in Bosga-Stork et al. (2011). In short it is based on the fact that large-amplitude arm movements tend to be performed at low frequencies by means of shoulder and elbow rotations, whereas small-amplitude arm movements tend to be performed at higher frequencies by means of wrist and finger rotations (Rosenbaum, Slotta, Vaughan \& Plamondon, 1991; Vaughan, Rosenbaum, Diedrich, \& Moore, 1996). In a task where the participants are forced to depart from these movement patterns (e.g., to produce fast shoulder movements or slow wrist rotations), they cannot rely on these intrinsic amplitudefrequency relationships and instead have to activate less natural, possibly more attention-demanding control regimes (cf. Zelaznik, Spencer \& Ivry, 2002; Bosga et al., 2005).

To capture the efficiency with which the children changed their movementparameter errors from cycle to cycle, we determined the ratio of the incidence of double-parameter changes that were in line with the inverse relationship between amplitude and frequency $\left(\mathrm{A}^{+} \mathrm{F}^{-}\right.$and $\left.\mathrm{A}^{-} \mathrm{F}^{+}\right)$over the incidence of parameter changes that were at odds with the inverse amplitude-frequency relationship $\left(\mathrm{A}^{+} \mathrm{F}^{+}\right.$ and $\left.\mathrm{A}^{-} \mathrm{F}^{-}\right)$. A ratio larger than 1.0 reflected efficient corrections of movementparameter errors. In the remainder of this article we refer to this ratio as the efficiency index.

Statistical Evaluation Two repeated-measures ANOVAs on the errors of the realized amplitudes and frequencies separately were performed. For the amplitudes we used a two-factor within subjects-design consisting of Age (7, 8, 9 years) and Instructed Amplitude (3, 6, 9 and $12 \mathrm{~mm}$ ), for the realized frequencies we used a design consisting of Age (7, 8, 9 years) and Instructed Frequency (1, 2, $3 \mathrm{~Hz}$ ). 
The degree to which the inverse relationship between movement amplitude and frequency was reflected in the average performance of the participants was evaluated in two separate repeated measure ANOVAs. The first ANOVA concerned the realized frequencies as a function of instructed amplitudes using a two-factor within-subject design consisting of Age (7, 8, 9 years) and Amplitude (3, 6, 9, and $12 \mathrm{~mm}$ ). The second ANOVA was directed at the realized amplitudes as a function of the imposed frequency. Again, we used a two-factor within-subject design consisting of Age (7, 8, 9 years) and Frequency (1, 2, $3 \mathrm{~Hz})$.

The degree to which the inverse relationship between movement amplitude and frequency was exploited while settling in into the loop-writing task was analyzed by evaluating the efficiency index (see Method section) by means of a univariate repeated-measures ANOVAs, using a full-factorial design consisting of the two within-subject variables Age (7, 8, 9 years) and Cycle (second, third, and fourth).

For all ANOVAs held that if the Mauchly test of sphericity indicated a violation of the normal data distribution, the Greenhouse-Geisser corrected F and p-values were chosen to evaluate within-subject effects, but to facilitate legibility we report the uncorrected dfs of the F-values. The critical value for statistical significance was set at .05. An a value of .10 was taken to reflect a weak developmental trend. Two-tailed $t$ tests $(\alpha=.05)$ were applied for post hoc age comparisons.

\section{Results}

\section{Global Task Performance}

The children were able to follow the amplitude instructions of 3, 6, 9, and $12 \mathrm{~mm}$ quite accurately $(\mathrm{F}[3.93]=955.14, p=.000$. Post hoc analyses (Scheffé's tests) showed that the realized amplitudes differentiated in four different categories $(0.5$, $-0.43,-1.36$, and $-2.27 \mathrm{~mm}$ ), which implies that the children's accuracy decreases with width.

The left panel of Figure 3 shows the constant error of amplitude of the children. The main effect of Age was significant, $\mathrm{F}(2,62)=5.3, p=.01$, the interaction between Age and Amplitude, however, was not significant $(\mathrm{F}[2,186]<1, p=.076)$. At ages 7, 8 and 9, the children displayed overshoots of the 3-mm instructed amplitude $(+.67,+.46,+.36 \mathrm{~mm}$, respectively), small undershoots of the 6 -mm instructed amplitude $(-.34,-.47$ and $-.49 \mathrm{~mm}$, respectively), and larger undershoots of the 9 and 12-mm instructed amplitudes $(-1.43,-1.28,-1.36 \mathrm{~mm}$, and $-2.57,-1.97$, $-2.28 \mathrm{~mm}$, respectively).

The children were also able to reproduce the frequency instructions of 1,2 , or $3 \mathrm{~Hz}(\mathrm{~F}[2,62]=492.82, p=.000)$. Post hoc analyses (Scheffé tests) showed that the realized frequencies differentiated in three different categories. At ages 7, 8 and 9, the instructed frequencies of 1 and $2 \mathrm{~Hz}$ were produced with small overshoots and undershoots $(1 \mathrm{~Hz}:+.13,+.12,+.11 \mathrm{~Hz}$, respectively; $2 \mathrm{~Hz}:-.10,-.05,+.01 \mathrm{~Hz}$, respectively) but the $3-\mathrm{Hz}$ frequency was reproduced with systematic undershoots $(-.60,-.35,-.26 \mathrm{~Hz})$.

The constant error of frequency is shown in Figure 3 (right panel). The main effect of Age was significant, $\mathrm{F}(2,62)=21.43, p=.000$, as was the interaction between Age and Frequency however was not significant $(\mathrm{F}[4,124]=11.04, p=.000)$. 
Generally, these results show that the children were able to perform our task as instructed, with efficiency increasing with age.

\section{Amplitude-Frequency Relationships}

The left panel of Figure 4 displays the realized frequency as a function of Age and Imposed Amplitude. The Age effect was statistically significant $(\mathrm{F}[2,62]=13.74$, $p=.000)$, as was the effect of Amplitude $(\mathrm{F}[3,93]=7.80, p=.001)$. There was no interaction between Age and Imposed Amplitude $(\mathrm{F}[6,186]<1, p=.663)$.

The realized amplitude as a function of Age and Imposed Frequency is displayed in the right panel of Figure 4. Here the Age effect was not statistically significant $(\mathrm{F}[2,62]<1, \mathrm{~ns})$, nor was the effect of Frequency $(\mathrm{F}[2,62]=3.491, p=.056)$, although there was a weak trend toward significance. There was a significant interaction between Age and Imposed Frequency $(\mathrm{F}[4,124]=3.910, p=.018)$. The distribution showed a U-shaped profile (the quadratic trend was significant) with amplitudes somewhat lower for the middle frequency.

Generally, these results show that the children were sensitive to the inverse relationship between movement amplitude and frequency even though their response to imposed frequencies was slightly more complicated than to changing amplitude requirements.

\section{Error Analyses}

Single-Parameter Errors: Amplitude. Figure 5 (left panel) displays the absolute error of the realized amplitude as a function of Age and Cycle. The Age effect was statistically significant $(\mathrm{F}[2,62]=11.37, p=.000)$ as was the effect of Cycle $(\mathrm{F}[2,62]=79.58, p=.000)$. The analysis revealed a significant interaction between Age and Cycle $(\mathrm{F}[4,124]=5.25, p=.008)$. These results show that the children were able to settle quickly into the task, leveling out at a stable and acceptable spatial performance in only two cycles. The ability to realize the required amplitudes improved over the years.

Single-Parameter Errors: Frequency. Figure 5 (right panel) shows the absolute error of the realized frequency as a function of Age and Cycle. The Age effect was statistically significant $(\mathrm{F}[2,62]=9.21, p=.000)$ as was the effect of Cycle $(\mathrm{F}[2,62]$ $=348.00, p=.000)$. The analysis revealed a significant interaction effect between Age and Cycle $(\mathrm{F}[4,124]=4.68, p=.007)$.

These results show that the children were able to settle quickly into the task, leveling out at a stable and acceptable performance in only two cycles. Their reproduction of the imposed frequency marginally (but significantly) improved with age.

Double-Parameter Errors: Amplitude and Frequency. Figure 6 depicts the results regarding the efficiency index (see Method section) revealing a statistically significant developmental trend. The main effect for Age was $\mathrm{F}(2,62)=3.639$, $p=.043$. Cycle proved statistically significant $(\mathrm{F}[2,62]=56.556, p=.000)$ but there was no interaction between Age and Cycle $(\mathrm{F}[4,124]<1$, ns). Overall, the efficiency index decreased with age: for 7 years $(\mathrm{M}=2.15, S D=0.76)$, for 8 years $(\mathrm{M}=1.95, S D=0.60)$ and for 9 years $(\mathrm{M}=1.88, S D=0.51)$. The index changed significantly between the ages 7 and 8 (paired $t[95]=3.531, p=.013$ ), but was not significantly different for ages 8 and 9 . The index also decreased within the first 


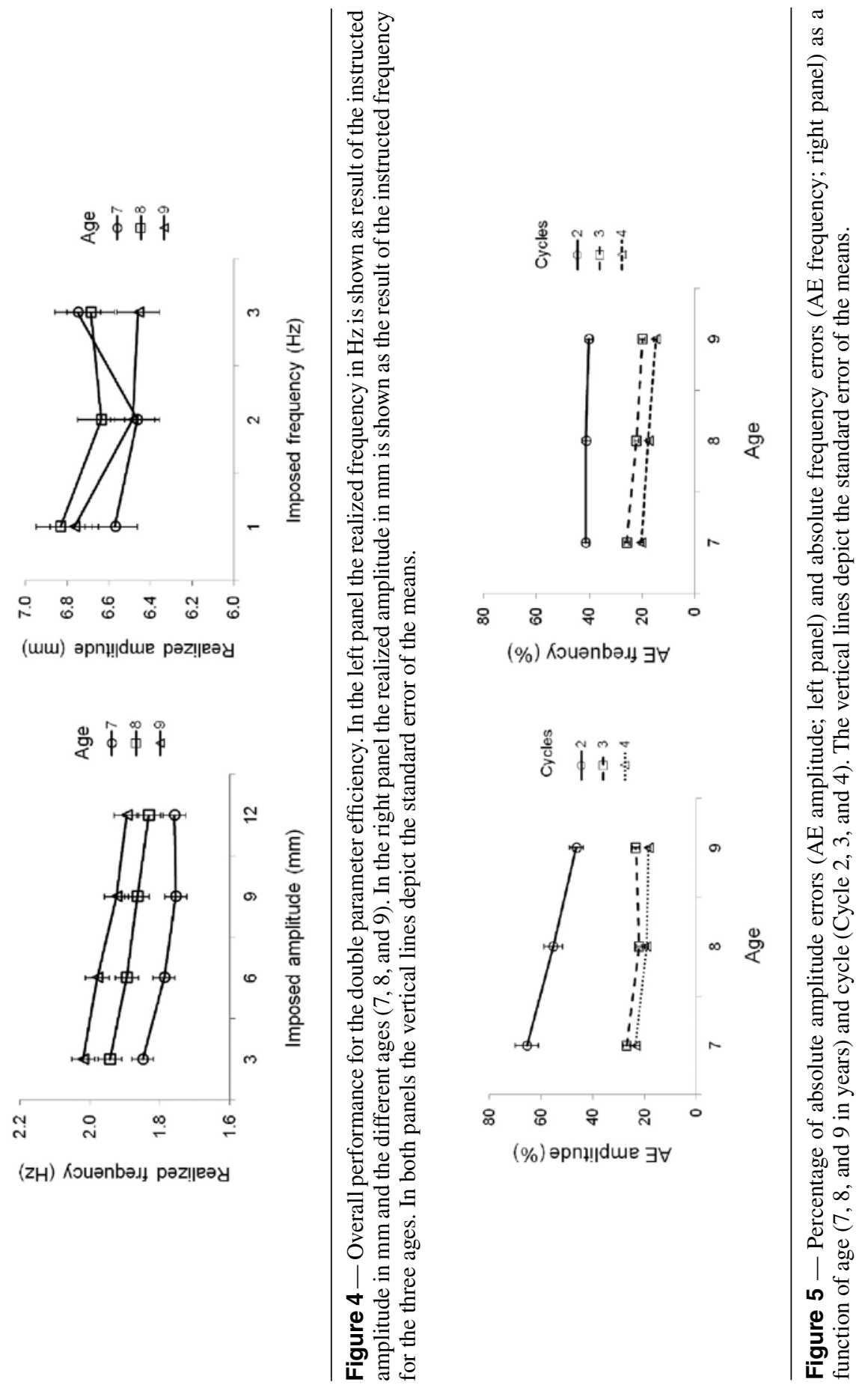




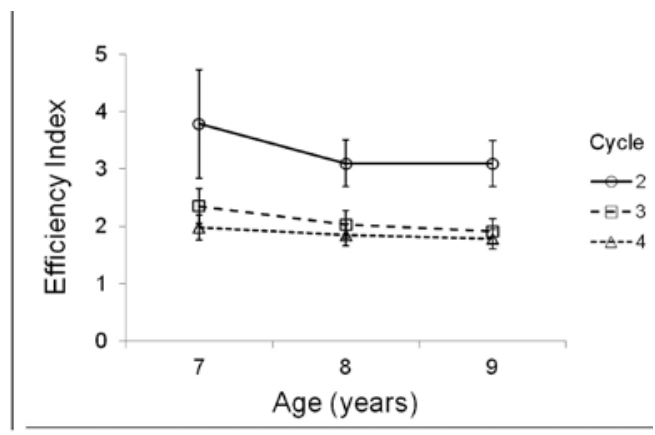

Figure 6 - The efficiency index as a function of age (7, 8, and 9 years) and cycle (Cycle 2,3 , and 4). The efficiency index is defined as the ratio of biomechanical- over intentional parameter error changes. When the ratio is $>1.0$, the error correction is in line with the inverse relationship between amplitude and frequency; see text). The vertical lines depict the standard error of the means.

three cycles (Cycle 2: $\mathrm{M}=2.34, S D=0.90$; Cycle 3: $\mathrm{M}=1.88, S D=0.57$; Cycle 4: $\mathrm{M}=1.76, S D=0.44)$ where the largest difference occurred between Cycles 2 and 3 (paired $t[95]=4.997, p=.000$ ) and only a weak tendency to significance was found between Cycles 3 and 4 (paired $t[95]=1.944, p=.055$ ).

These results show that the efficiency index remained fairly stable over the years and was most pronounced during the early cycles of our loop-writing task.

\section{Discussion}

\section{Research Question}

The present longitudinal study analyzed the efficiency with which primary school children at age 7, 8, and 9 try to simultaneously meet spatial and temporal movement instructions while settling into a loop-writing task. We analyzed (i) how well the children reproduced the instructed amplitudes and frequencies, (ii) whether their behavior reflected the inverse amplitude-frequency relationship, and (iii) the extent to which they exploited this relation when correcting errors from cycle to cycle while settling into the task. Before discussing our main findings it must be noted that we did not expect qualitative changes in performance as, for example, reported by Peper and Beek (1998) since we studied effects of a fairly small, educationally relevant range of parameter changes.

\section{Developing Efficiency}

As regards the realized amplitudes the primary school children displayed systematic undershoots of up to $25 \%$ of the instructed loop sizes, thus reflecting the general tendency of people to perform undershoots of spatial targets. Such a tendency has been attributed to energy containment (Elliot, Hansen, Mendoza, \& Tremblay, 2004). These findings are in line with the results found by Vinter and Mounoud (1991). 
Among the four amplitudes tested the 3-mm amplitude stood out, since at all ages the children overshot this amplitude. Apparently, the smallest amplitude required too much movement precision for the children to perform accurately (Trap-Porter, Gladden, Hill, \& Cooper, 1983). When asked for their opinion, they expressed that the 6-mm amplitude was the easiest amplitude to produce. Developmentally, the children systematically improved in amplitude reproduction with age.

It seems reasonable to expect older children to realize the required loop amplitudes more efficiently than younger children because between the ages of 7 and 9 their hands grow larger. However, we do not consider this line of reasoning correct. The argument may hold if the loop-writing task would be performed by the hand only, i.e., through wrist and finger movements but earlier studies have shown that even adults involve prosupinations, forearm rotations and (moderate) shoulder excursions in loop writing tasks involving amplitude sizes as applied here (Meulenbroek, Thomassen, van Lieshout, \& Swinnen, 1998; Meulenbroek, Rosenbaum, Thomassen, \& Schomaker, 1993). Furthermore, in the present loop-writing task we did not impose constraints on limb-segment involvement. We therefore attribute the age-dependent improvement in amplitude reproduction to increased movement efficiency.

As concerns the realized movement frequencies the children performed the $2-\mathrm{Hz}$ frequency most accurately. The $1-\mathrm{Hz}$ frequency was performed too fast and the $3-\mathrm{Hz}$ frequency too slow. These results replicate earlier findings concerning preferred movement frequencies in cyclical drawing behavior in adults (Meulenbroek et al., 1993) and are compatible with models capitalizing on the role of preferred amplitudes and frequencies and cognitive efficiency constraints in movement selection (Rosenbaum et al., 1991). When asked, the children were upfront in their opinion that the $2-\mathrm{Hz}$ speed instruction was the easiest to comply with. As regards development, the $3-\mathrm{Hz}$ frequency stood out because it showed an error reduction of $33 \%$ between the ages of 7 and 9 . In general, the children thus became more skilled in attaining the highest, acoustically instructed frequency.

Considering the way in which they responded to the combined speed-amplitude instructions, the children generally showed sensitivity to the natural inverse relationship between the two parameters. As they grew older the sensitivity became more pronounced. At nine years of age, imposing larger amplitudes elicited lower frequencies and instructing higher frequencies elicited smaller amplitudes, as expected.

Theoretically, the improved performance we presently observed as the children aged between 7 and 9 years might be related to the development of abilities such as transitivity (Piaget \& Inhelder, 1965; Pears \& Bryant, 1990). The ability to understand the ordinal scaling of elements on the basis of one of their geometrical properties, e.g., length, height etc.-i.e., being able to recognize and apply transitive relations - presupposes an internal representation of such parameters. Here, we presume that our participants have the parameters movement amplitude and frequency internally represented while performing our loop-writing task. In first grade the children's age range was between 6.3 and 7.5, an age in which the possibility of still lingering disorganization between temporal and spatial similarities in movement performance cannot be excluded (see also Vinter \& Mounoud, 1991).

Next we turn to how quick the children settled into the task as far as meeting the amplitude and frequency constraints concerned. With respect to movement ampli- 
tude, the children used the second movement cycle to explore the task requirements and settled into the correct amplitude occurred in the third and fourth movement cycles. Here a clear developmental change was found. Adaptations to the frequency task requirements were comparable. The children again primarily used the second movement cycle to settle into frequency requirements. The developmental trend was less pronounced than observed in the amplitude data. In sum, the first part of our prediction where we assumed that children would be able to settle quickly into task requirements as reflected by decreasing performance errors, was confirmed. The second part in which we predicted improvement with age was only confirmed for the amplitude requirements, and then specifically in the second cycle.

The movement efficiency increases that we did observe as the children aged can be attributed to several factors. First, with age children are likely to develop better sensorimotor representations of learned tasks (e.g., by generating internal models), which in turn allows the central nervous system to comply with the spatiotemporal demands of the task better. Second, attentional capacity should not be ruled out as potential factor. The combination of visual and auditory stimuli in our experimental task may have imposed relatively strong attentional demands on our participants. In their longitudinal study of attentional capacity in 6-12 year-old children, Robbers et al. (2011) identified attentional problems in $18 \%$ of their subject group. Considering that our participants were typically developing primary school children, a similar percentage could be estimated to have had attentional deficits, a matter that we did not assess separately. Consequently, at age 7 the children might have been particularly prone to fatigue while trying to keep up attention for $45 \mathrm{~min}$. This might explain the larger number of error trials observed at this age. A third factor involved might be that the children performed the loop-writing task in a more closed-loop fashion at age 7 whereas at age 9 they may have managed to adopt more open-loop control strategy. To determine the extent to which the age-related movement efficiency increase can be attributed to an improved capability to exploit the biomechanics of their developing motor system such as inertia, interaction torques and energy costs, future research should include kinetic analyses and estimate to what extent the motion of the hand is driven by concentric and eccentric muscle contraction. Another approach would be to look into smoothness of acceleration or deceleration profiles, presuming that skilled performers are expected to be less jerky.

\section{Efficiency in Cycle-to-Cycle Parameter Changes}

Our main prediction was an age-dependent increase in the efficiency with which the children would exploit the inverse amplitude-frequency relationship when correcting movement errors while settling in into our loop-writing task. Even though marginal, we found, contrary to our expectations, an age-dependent decrease in this error-correction efficiency. This unexpected result may seem uninformative but in the light of developmental theories, the almost constant efficiency of cyclical movement behavior in an educationally relevant task is in our view remarkable (Harcum, 1990). Indeed, whether motor development between 7 and 9 years of age is linear or nonlinear, the observed constancy reflects an inability to increase performance-monitoring efficiency, which in our view must reflect an important constraint on skill acquisition. We will return to this aspect below. 
In the current study we found that the incidence of parameter changes that were in line with the inverse amplitude-frequency relationship amounted to $61 \%$. In a study using the same paradigm involving adults, Bosga et al., (2005) reported an incidence of $41 \%$ of such parameter changes. This relatively low incidence of this specific category of parameter changes was probably due to the more stringent timing constraints of the adult task (where 4 and $5 \mathrm{~Hz}$ movement frequencies were also tested).

\section{Relation to Educational Settings}

The absence of an efficiency increase in error correction as the children aged between 7 and 9 years might be due to various educational factors. For example, the children may, at this stage of their development, still be occupied with discovering, through trial and error, how fast and accurate they need to be to achieve the task goals but not yet actually be able to combine speed and accuracy properly. An alternative cause for our findings might be that as the children grew older, they (i) became eager to show their potential in test situations such as used here, and (ii) the amplitude targets might have been more easily attainable than the frequency targets, e.g., because vision dominates audition (see also below). In case of such bias for spatial processes, the sensitivity to the inverse relationship between the amplitude and frequency might be compromised. In a similar vein, the handwriting curricula in the Netherlands emphasize accuracy of letterform within a prescribed space starting in first grade, with lineation height around $4.5 \mathrm{~mm}$. In second grade speed exercises are added, still within a prescribed lineation height, now of 3.5 $\mathrm{mm}$. In third grade this is maintained, with a lineation size of $2.5 \mathrm{~mm}$, so it is not unthinkable that the children implicitly try to comply with their teachers' wishes. Trap-Porter et al. (1983) found space size and accuracy in second and third graders to be related. Cursive writing is enhanced when large-spaced writing paper is used in these grades. Although the research concerned manuscript writing, a similar result was found for first graders by Kau-To Leung, Trablas, Hill, \& Cooper (1979). In school settings, spatial restrictions are indeed more important in handwriting curricula than temporal constraints (Graham \& Weintraub, 1996). As proposed, such emphases on spatial task goals may prevent children from flexibly changing their movement parameters while monitoring and correcting their performance errors. The just described educational factors may also be at odds with sensorimotor developmental processes.

Theories of sensorimotor learning state that the amount of cognitive control diminishes where experience and training increase (Gentile, 2000; Fitts \& Posner, 1967). When cognitive control is gradually replaced by automaticity the learner must increase consistency and efficiency until the most economic coordination pattern is reached. An overall increase of biomechanically efficient error correction, as would be expected to accompany reduced cognitive control, was currently not found. Potentially, because the children had to start the loop-writing task while adjusting to a metronome, thus enforcing an explicitly planned and cognitively controlled action. Clearly at this age children are not yet fully able to efficiently combine auditory, visual and motor information. When older and more experienced in handwriting, eight and nine year-old children are probably more able to use feedforward processes as an anticipatory error-correction mechanism. 
They are trained to maintain the vertical amplitude of their script between the horizontal lineation and probably are also able to treat auditory and visual stimuli as one compound stimulus, where amplitude requirements are more important than temporal cues. In their study on dissociation of explicit and implicit timing in repetitive tapping and drawing movements, Zelaznik, Spencer, and Ivry (2002) concluded that the timing of movement initiation is an explicit process, whereas the timing of movement duration is an implicit process. They further concluded that once the movement is started temporal control might emerge as a function of higher-level conceptualization of the task change to an emergent property of other control processes.

\section{Conclusion}

The present study provided a window into adaptation and development using a loop-writing task in which multiple goals needed to be achieved simultaneously. A cohort of primary school children was followed at 7, 8, and 9 years of age with the purpose to record the changes in movement efficiency in childhood. In contrast to our expectations, we observed relative constancy as regards the exploitation of the inverse relationship between amplitude and frequency while correcting parameter errors. The lack of exploiting amplitude-frequency relationships during error correction may be due to the emphasis on spatial accuracy in current handwriting educational practices in the Netherlands, which makes abundant use of lineation combined with global speed instructions. A crucial question that still remains concerns interindividual differences and, more importantly, the relevance of motor efficiency and flexibility for school performance.

\section{References}

Bosga-Stork, I.M., Bosga, J., \& Meulenbroek, R.G.J. (2011). Intentional Control and biomechanical exploitation in preparatory handwriting. Human Movement Science, 30, 687-697. PubMed doi:10.1016/j.humov.2010.06.007

Bosga, J., Meulenbroek, R.G.J., \& Rosenbaum, D.A. (2005). Deliberate control of continuous motor performance. Journal of Motor Behavior, 37, 437-446. PubMed doi:10.3200/ JMBR.37.6.437-446

Dusing, S.C., \& Harbourne, R.T. (2010). Variability in postural control during infancy: Implications for development, assessment, and intervention. Physical Therapy, 90(12), 1838-1849. PubMed doi:10.2522/ptj.2010033

Elliott, D., Hansen, S., Mendoza, J., \& Tremblay, L. (2004). Learning to optimize speed, accuracy, and energy expenditure: A framework for understanding speed-accuracy relations in goal-directed aiming. Journal of Motor Behavior, 36, 339-351. PubMed doi:10.3200/JMBR.36.3.339-351

Fitts, P.M. (1954). The information capacity of the human motor system in controlling the amplitude of movement. Journal of Experimental Psychology, 47, 381-391. PubMed doi:10.1037/h0055392

Fitts, P.M., \& Posner, M.I. (1967). Human performance (pp. 8-25). Belmont, CA: BrooksCole.

Gentile, A.M. (2000). Skill Acquisition: Action, movement and neuromotor processes. In J.H. Carr \& R.B. Shepard (Eds.), Movement Science: Foundation for physical therapy in rehabilitation (2nd ed., pp. 111-187). Rockvill, MD: Aspen. 
Graham, S., \& Weintraub, N. (1996). A review of handwriting research: Progress and prospects from 1980 to 1994. Educational Psychology Review, 8, 7-87. doi:10.1007/ BF01761831

Harcum, E.R. (1990). Distinction between tests of data or theory: Null versus disconfirming results. The American Journal of Psychology, 103, 359-366. doi:10.2307/1423215

Hazeltine, E., \& Wifall, T. (2011). Searching working memory for the source of dual-task costs. Psychological Research, 75, 466-475. PubMed doi:10.1007/s00426-011-0343-6

Kau-To Leung, E., Trablas, P.V., Hill, D.S., \& Cooper, J.O. (1979). Space size and accuracy of first grade students' manuscirpt writing. The Journal of Educational Research, 73, 79-81.

Laquaniti, F., Terzuelo, C., \& Viviani, P. (1984). Global metric properties and preparatory processes in drawing movements. In S. Kornblum \& J. Requin (Eds.), Preparatory states and processes (pp. 357-370). Hillsdale, New York: Erlbaum.

Magill, R. (2011). Defining and assessing learning. In R.A. Magill (Ed.), Motor learning and control: Concepts and applications (9th ed.; pp. 248-252). New York: McGraw-Hill.

Meulenbroek, R.J.G., \& Van Galen, G.P. (1988). The acquisition of skilled handwriting: discontinuous trends in kinematic variables. In A.M. Colley \& J.R. Beech (Eds.), Cognition and action in skilled behavior (pp. 273-281). North Holland: Elsevier Science Publishers B.V.

Meulenbroek, R.G., Rosenbaum, D.A., Thomassen, A.J., \& Schomaker, L.R. (1993). Limbsegment selection in drawing behavior. Quarterly Journal of Experimental Psychology, 46, 273-299. PubMed

Meulenbroek, R.G., Thomassen, A.J., van Lieshout, P.H., \& Swinnen, S.P. (1998). The stability of pen-joint and interjoint coordination in loop writing. Acta Psychologica, 100, 55-70. PubMed doi:10.1016/S0001-6918(98)00025-0

Morris, C. (2009). Measuring participation in childhood disability: how does the capability approach improve our understanding. Developmental Medicine and Child Neurology, 51, 92-94. PubMed doi:10.1111/j.1469-8749.2008.03248.x

Newell, K.M., \& van Emmerik, R.E.A. (1989). The acquisition of coordination: Preliminary analysis of learning to write. Human Movement Science, 8, 17-32. doi:10.1016/01679457(89)90021-3

Newell, K.M. (1986). Constraints on the development of coordination. In M.G. Wade \& H.T.A. Whiting (Eds.), Motor skill acquisition in children: Aspects of coordination and control (pp. 341-360). Amsterdam: Martinus Nijhoff Publishers.

Pears, R., \& Bryant, P. (1990). Transitive inferences by young children about spatial position. The British Journal of Psychology, 81, 497-510. doi:10.1111/j.2044-8295.1990. tb02375.x

Piaget, J., \& Inhelder, B.A. (1965). The child's conception of space. London: Routledge $\&$ Kegan Paul.

Peper, C.E., \& Beek, P.J. (1998). Are frequency-induced transitions in rhythmic coordination mediated by a drop in amplitude? Biological Cybernetics, 79, 291-300. PubMed doi:10.1007/s004220050479

Robbers, S.C., van Oort, F.V., Polderman, T.J., Bartels, M., Boomsma, D.I., Verhulst, F.C., . . Huizink, A.C. (2011). Trajectories of CBCL Attention Problems in Children. European Child \& Adolescent Psychiatry, 20, 419-427. PubMed doi:10.1007/s00787011-0194-0

Rosenbaum, D.A., Slotta, J.D., Vaughan, J., \& Plamondon, R. (1991). Optimal movement selection. Psychological Science, 2, 86-91. doi:10.1111/j.1467-9280.1991.tb00106.x

Shumway-Cook, A., \& Woollacott, M.H. (2007). Motor Control: Translating research into clinical practice (3rd ed., pp. 511-512). Philadelphia: Wolters Kluwer Health/Lippincott Williams \& Wilkins.

Thelen, E. (2005). Dynamic systems theory and the complexity of change. Psychoanalytic Dialogues, 15, 255-283. doi:10.1080/10481881509348831 
Trap-Porter, J., Gladden, M.A., Hill, D.S., \& Cooper, J.O. (1983). Space size and accuracy of second and third grade students' cursive handwriting. The Journal of Educational Research, 76, 231-233.

Vaughan, J., Rosenbaum, D.A., Diedrich, F.J., \& Moore, C.M. (1996). Cooperative selection of movements: The optimal selection model. Psychological Research, 58, 254-273.

Vinter, A., \& Mounoud, P. (1991). Isochrony and accuracy of drawing movements in children: effects of age and context. In J. Wann, A.M. Wing, \& N. Sõvik (Eds.), Development of graphic skills (pp. 113-134). London: Academic Press.

Viviani, P., \& Terzuolo, C.A. (1980). Space-time invariance in learned motor skills. In G.E. Stelmach \& J. Requin. (Eds.), Tutorials in motor behavior (Vol. 1, pp. 525-533). Amsterdam: North Holland.

Viviani, P., \& McCollum, G. (1983). The relation between linear extent and velocity in drawing movements. Neuroscience, 10, 211-218. PubMed doi:10.1016/03064522(83)90094-5

von Hofsten, C. (2004). An action perspective on motor development. Trends in Cognitive Sciences, 8, 266-272. PubMed doi:10.1016/j.tics.2004.04.002

Zelaznik, H.N., Spencer, R.M.C., \& Ivry, R.B. (2002). Dissociation of explicit and implicit timing in repetitive tapping and drawing movements. Journal of Experimental Psychology. Human Perception and Performance, 28, 575-588. PubMed doi:10.1037/00961523.28.3.575

\section{Footnotes}

${ }^{1}$ In a separate analysis involving all cycles, we did not find a different pattern of results. 